Uso de índice de vegetação para caracterizar a mudança no uso do solo em Coari-AM Adriano Pereira Guilherme, Andréa Baima dos Santos Mota, Deniz dos Santos Mota, Nadja Gomes Machado, Marcelo Sacardi Biudes

\title{
USO DE ÍNDICE DE VEGETAÇÃO PARA CARACTERIZAR A MUDANÇA NO USO DO SOLO
} EM COARI-AM

\section{Use of vegetation index to characterize the land use change in Coari - AM}

\author{
Adriano Pereira Guilherme \\ Universidade Federal do Amazônas, Coari, Amazônas, Brasil. \\ adrianopgpg@gmail.com \\ Andréa Baima dos Santos Mota \\ Instituto Federal do Amazônas, Coari, Amazônas, Brasil. \\ andrea_baima@hotmail.com \\ Deniz dos Santos Mota \\ Universidade Federal do Amazônas, Coari, Amazônas, Brasil. \\ dmotaufam@gmail.com \\ Nadja Gomes Machado \\ Instituto Federal de Mato Grosso, Cuiabá, Mato Grosso, Brasil. \\ nadja.machado@blv.ifmt.edu.br \\ Marcelo Sacardi Biudes \\ Universidade Federal de Mato Grosso, Cuiabá, Mato Grosso, Brasil. \\ marcelo@fisica.ufmt.br
}

Artigo recebido em 08/09/2015 e aceito para publicação em 01/07/2016

RESUMO: O município de Coari apresentou transformação significativa no uso e ocupação do solo nas últimas décadas, parte causada provavelmente pela atividade de prospecção e exploração de óleo e gás natural, e parte devido ao aumento populacional do município. Os índices de vegetação possibilitam a caracterização e quantificação de parâmetros biofísicos da superfície, pois reduzem a dimensão das informações multiespectrais fornecidas pelos satélites. Sendo assim, o objetivo deste trabalho foi estudar a mudança do uso e ocupação do solo no município de Coari-AM por meio do índice de vegetação da diferença normalizada (NDVI) calculado a partir de imagens Landsat 5 TM em 1986 e 2009. Foram utilizadas duas imagens obtidas pelo satélite Landsat $5 \mathrm{TM}$, compostas de sete bandas espectrais e disponibilizadas no site do Instituto de Pesquisas Espaciais - INPE. A classificação quanto ao uso e ocupação do solo foi realizada a partir do NDVI obtido da imagem Landsat 5 TM e definidas quatro classes: área urbana ou solo exposto, floresta densa, clareiras e água. Os resultados obtidos possibilitaram visualizar as mudanças no uso e ocupação do solo da região de Coari. Houve um aumento de solo exposto/área urbana e diminuição da área de floresta densa, demostrando o avanço da atividade antrópica sobre a região nas duas últimas décadas.

Palavras chave: sensoriamento remoto; cobertura vegetal; desmatamento; urbanização. 
Uso de índice de vegetação para caracterizar a mudança no uso do solo em Coari-AM

Adriano Pereira Guilherme, Andréa Baima dos Santos Mota, Deniz dos Santos Mota, Nadja Gomes Machado, Marcelo Sacardi Biudes

ABSTRACT: The municipality of Coari showed significant transformation in land use and occupation in recent decades, probably caused by the activity of prospecting and exploration of oil and natural gas, as well as due to its population increase. The vegetation indices enable characterization and quantification of biophysical parameters of the surface, once they reduce the size of the multispectral information provided by satellites. Therefore, the aim of this study was to study changes in land use and occupation in Coari-AM through the normalized difference (NDVI) vegetation index calculated from Landsat 5 TM in 1986 and 2009. Two images obtained by satellite Landsat 5 TM were used, composed of seven spectral bands and available by Space Research Institute site - INPE. Classification of land use and occupation was held from the NDVI obtained from Landsat 5 TM image and defined four classes: urban area or exposed soil, dense forest, clearings and water. The results enabled to view changes in the land use and occupation in Coari. There was an increase of exposed soil / urban area and decreasing the area of dense forest, showing the advancement of human activity on the region in the last two decades.

Keywords: remote sensing; vegetation cover; deforestation; urbanization.

\section{INTRODUÇÃO}

A partir da década de 1980, a região Norte do Brasil tem apresentado aumento no número da população. A taxa de aumento da população variou entre as décadas seguintes, mas foi a partir de 1996 que o governo estadual impôs uma política de governo com vista ao reordenamento e dinamização da região (MOURÃO, 2003). O município de Coari-AM, situado às margens do rio Urucu na bacia do Solimões, sedia uma das principais áreas produtoras de petróleo e gás da Amazônia, denominada de Província Petrolífera de Urucu. Em 1988, deu-se início a produção comercial de petróleo e gás natural. A partir de então, Coari-AM apresentou transformações significativas no uso e ocupação do solo com um crescimento demográfico acelerado de 38.313 habitantes em 1992 para 78.982 habitantes em 2010 (IBGE, 2011). A economia do município tem sido impulsionada pela exploração de petróleo e gás natural, em contexto de elevado impacto ambiental (FERNISIDE, 2008) e problemas sanitários de grande magnitude, principalmente na malha urbana do município (ALMEIDA; SOUZA, 2008). Em 2008, o PIB de Coari era de R \$ 895,92 milhões, o segundo maior do estado do Amazonas (AMAZONAS, 2008), contrastando com um Índice de Desenvolvimento Humano (IDH) de médio desenvolvimento humano (de aproximadamente 0,63 ), o trigésimo terceiro do estado, com renda média mensal de R\$ 164,50 e per capita de R\$ 110,97 (MOURÃO et al., 2007).

Imagens de sensores orbitais, como o caso do satélite Landsat 5 TM (Thematic Mapper) têm sido muito utilizadas em estudos temporais de análise das mudanças da cobertura e uso do solo (FOODY, 2010; GÓMEZ et al., 2011; CUNHA et al., 2011). O satélite Landsat 5, da Série Landsat, que entrou em funcionamento em 01/03/1984 e foi desativado oficialmente em 05/06/2013. O satélite estava a $705 \mathrm{~km}$ acima da Terra e as informações eram recolhidas utilizando um mapeador temático com resolução espacial de 30 metros. O que tornou o Landsat 5 TM o mais importante dos satélites da série Landsat foi o fato dele ter sido projetado para atuar durante 3 anos, mas acabou operando por mais de 29 anos, sendo reconhecido pelo Guinness Book of Records como o mais antigo equipamento orbital de observação remota da história (AEROMAPA S/A, 2013).

O elevado tempo de operação do satélite Landsat 5 TM possibilita identificar, de forma contínua, tendências nos pixels das imagens geradas em função da mudança do uso do solo provocada durante décadas (KENNEDY et al., 2014). Outro aspecto das imagens Landsat 5 TM é a sua disponibilidade gratuita, o que possibilita estudos de baixo custo em escala regional/ global (HOSTERT et al., 2003).

Dentre as variáveis utilizadas para avaliação da mudança do uso do solo, os índices de vegetação têm sido mais utilizados. Os índices de vegetação possibilitam a caracterização e quantificação de parâmetros biofísicos de florestas, culturas agronômicas e mudanças provocadas no uso do solo, pois reduzem a dimensão das informações multiespectrais fornecidas pelos satélites (TUCKER, 1985). O índice de vegetação da diferença normalizada (do inglês Normalized 
Uso de índice de vegetação para caracterizar a mudança no uso do solo em Coari-AM Adriano Pereira Guilherme, Andréa Baima dos Santos Mota, Deniz dos Santos Mota, Nadja Gomes Machado, Marcelo Sacardi Biudes

Difference Vegetation Index - NDVI) é apontado como um dos mais aceitos índices para analisar a cobertura vegetal utilizando o sensoriamento remoto (HOLBEN et al., 2007), pois além do diagnóstico detalhado da fitomassa fotossinteticamente ativa, corrige os efeitos de sombras oriundas da elevação topográfica sobre a imagem (ELVIDGE; LYON, 1985; TUCKER, 1985). Diante disso, o objetivo deste trabalho foi estudar a mudança do uso e ocupação do solo no município de Coari-AM por meio do NDVI calculado a partir de imagens Landsat 5 TM. Neste trabalho foram verificadas as alterações no percentual de uso do solo exposto e zona urbana (aqui denominado apenas como solo exposto/cidade) e de floresta, em virtude da expansão demográfica entre 1986 e 2009.

\section{MATERIAL E MÉTODOS}

A área analisada está compreendida entre as coordenadas $63^{\circ} 06^{\prime} 26^{\prime \prime}$ e $63^{\circ} 16^{\prime} 05^{\prime \prime}$ de longitude oeste e $3^{\circ} 56^{\prime} 03^{\prime \prime}$ e $4^{\circ} 09^{\prime} 54^{\prime \prime}$ latitude sul, pertencendo ao município de Coari, Amazonas (Figura 1). A região fica aproximadamente no centro geográfico do estado, no coração da floresta amazônica. O clima é equatorial quente e úmido, correspondendo à classificação de Köppen ao tipo Ami (PEEL et al., 2007), com precipitação pluvial anual igual ou superior a $2.000 \mathrm{~mm}$ e com médias anuais de temperatura e umidade relativa de $27^{\circ} \mathrm{C}$ e $84 \%$, respectivamente. A zona urbana está localizada próxima ao Rio Solimões entre os Lagos Coari e Mamiá, com uma altitude média de $46 \mathrm{~m}$. Em 1932, Coari foi elevada à categoria de cidade e atualmente possui o segundo maior produto interno bruto do Amazonas. Essa posição deve-se à extração de petróleo e gás da base petrolífera de Urucu, que possui uma produção média de petróleo de mais de 50 mil barris e 10,36 mil metros cúbicos de gás natural por dia (PETROBRÁS, 2013).

Figura 1 - Localização do município de Coari, Amazonas, Brasil.

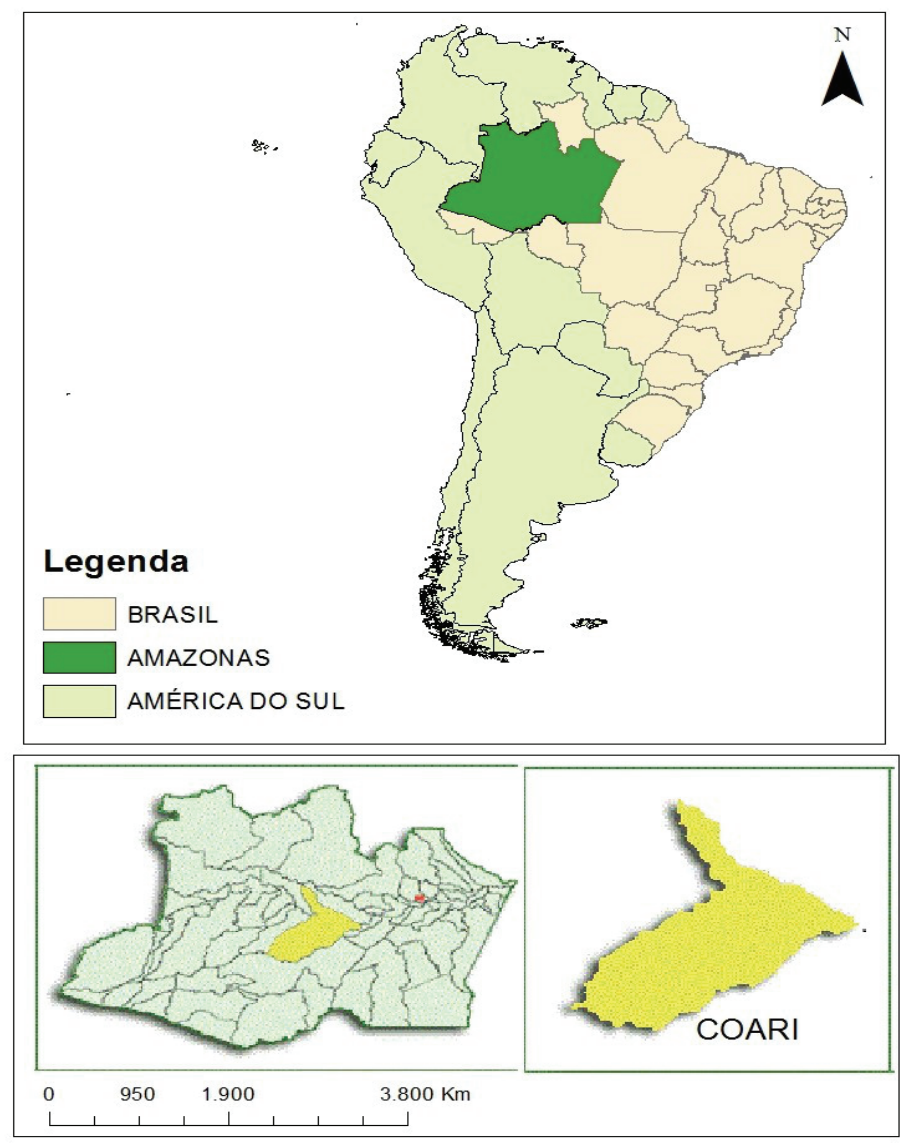

Org. dos autores. 
Uso de índice de vegetação para caracterizar a mudança no uso do solo em Coari-AM

Adriano Pereira Guilherme, Andréa Baima dos Santos Mota, Deniz dos Santos Mota, Nadja Gomes Machado, Marcelo Sacardi Biudes

Neste trabalho foram utilizadas duas imagens obtidas pelo satélite Landsat 5 TM, compostas de sete bandas espectrais. Essas imagens correspondem à órbita 233 e ao ponto 63, disponibilizadas no site do Instituto de Pesquisas Espaciais - INPE. Para a análise dessas imagens foram necessários alguns dados (fornecidos também pelo INPE) que podem ser verificados na Tabela 1.

Tabela 1 - Dia, hora e elevação solar do momento da passagem do satélite Landsat 5 TM.

\begin{tabular}{|c|c|c|}
\hline Dia & Horário GMT & Elevação do Sol \\
\hline $08 / 08 / 1986$ & $13 \mathrm{~h} 47 \mathrm{~min} 35 \mathrm{~s}$ & 47,1735 \\
\hline $07 / 08 / 2009$ & $14 \mathrm{~h} 14$ min $54 \mathrm{~s}$ & 52,8632 \\
\hline \multicolumn{2}{|c|}{ Org. dos autores. }
\end{tabular}

Cada banda espectral possui seus próprios coeficientes de calibração, propostos por Chander e Markham (2003). Efetuou-se a calibração radiométrica da imagem, que consiste na conversão do número digital (ND), número inteiro que varia de 0 a 255 , em radiância espectral monocromática $L_{\lambda \mathrm{i}}$ (medida em $\mathrm{W} \mathrm{m}^{-2} \mathrm{~mm}^{-1}$ ) e que, segundo Markham \& Baker (1987), é determinada pela equação (1) para as sete bandas refletivas.

$$
L_{\lambda i}=a_{i}+\left[\frac{\left(b_{i}-a_{i}\right)}{255}\right] . N D
$$

em que e são os coeficientes de calibração para a -ésima banda.

A refletância espectral monocromática de cada banda $\left(\rho_{\lambda \mathrm{i}}\right)$ é razão entre a radiação refletida pela superfície medida pelo satélite e a radiação solar incidente, definida pela equação (2) (ALLEN et al., 2002).

$$
\rho_{\lambda i}=\frac{\left(\pi L_{\lambda i}\right)}{\left(k_{\lambda i} \cos Z d_{r}\right)}
$$

em que $k_{\lambda \mathrm{i}}$ a irradiância solar espectral de cada banda no topo da atmosfera $\left(\mathrm{Wm}^{-2} \mathrm{~mm}^{-1}\right)$ e $d_{r}$ é o quadrado da razão entre a distância média Terra-Sol $\left(r_{0}\right)$ e a distância Terra-Sol $(r)$ em dado dia do ano. Segundo Iqbal (1983), pode ser calculada pela equação (3) em função do dia Juliano (DJ).

$$
d_{r}=1+0,033 \cos \left(\frac{2 \pi D J}{365}\right)
$$

O cálculo do índice de vegetação da diferença normalizada (NDVI) (equação 4), estabelecido por Tucker (1979), é obtido pela razão entre a diferença e a soma das refletividades do infravermelho próximo $\left(\rho_{I V}\right)$, referente à banda 4 (Infravermelho próximo), e do vermelho $\left(\rho_{V}\right)$, referente à banda 3 .

$$
N D V I=\frac{\left(\rho_{I V}-\rho_{V}\right)}{\left(\rho_{I V}+\rho_{V}\right)}
$$

Os resultados possíveis do NDVI estão compreendidos entre -1 e 1 , o qual indicará a cobertura vegetal da nossa área de estudo. Quanto maior o valor positivo do NDVI maior a presença de vegetação. A razão para o uso destas b andas específicas se deve ao fato da maior absorbância da cobertura vegetal estar na faixa do vermelho, que no caso é medido pelo Landsat 5 TM pela banda 4 do infravermelho próximo. (MENESES e ALMEIDA, 2012).

\section{RESULTADOS E DISCUSSÕES}

A Figura 2 representa as imagens da área de estudo em cor verdadeira, capturadas em 1986 e 2009, respectivamente. Em aproximadamente 20 anos, é visível o aumento das áreas de solo exposto/ cidade, que tiveram como principal causa provável a expansão do município e a exploração dos recursos naturais da região. 
Uso de índice de vegetação para caracterizar a mudança no uso do solo em Coari-AM Adriano Pereira Guilherme, Andréa Baima dos Santos Mota, Deniz dos Santos Mota, Nadja Gomes Machado, Marcelo Sacardi Biudes

Figura 2 - Imagens do satélite Landsat 5 TM do município de Coari - Amazonas/Brasil.
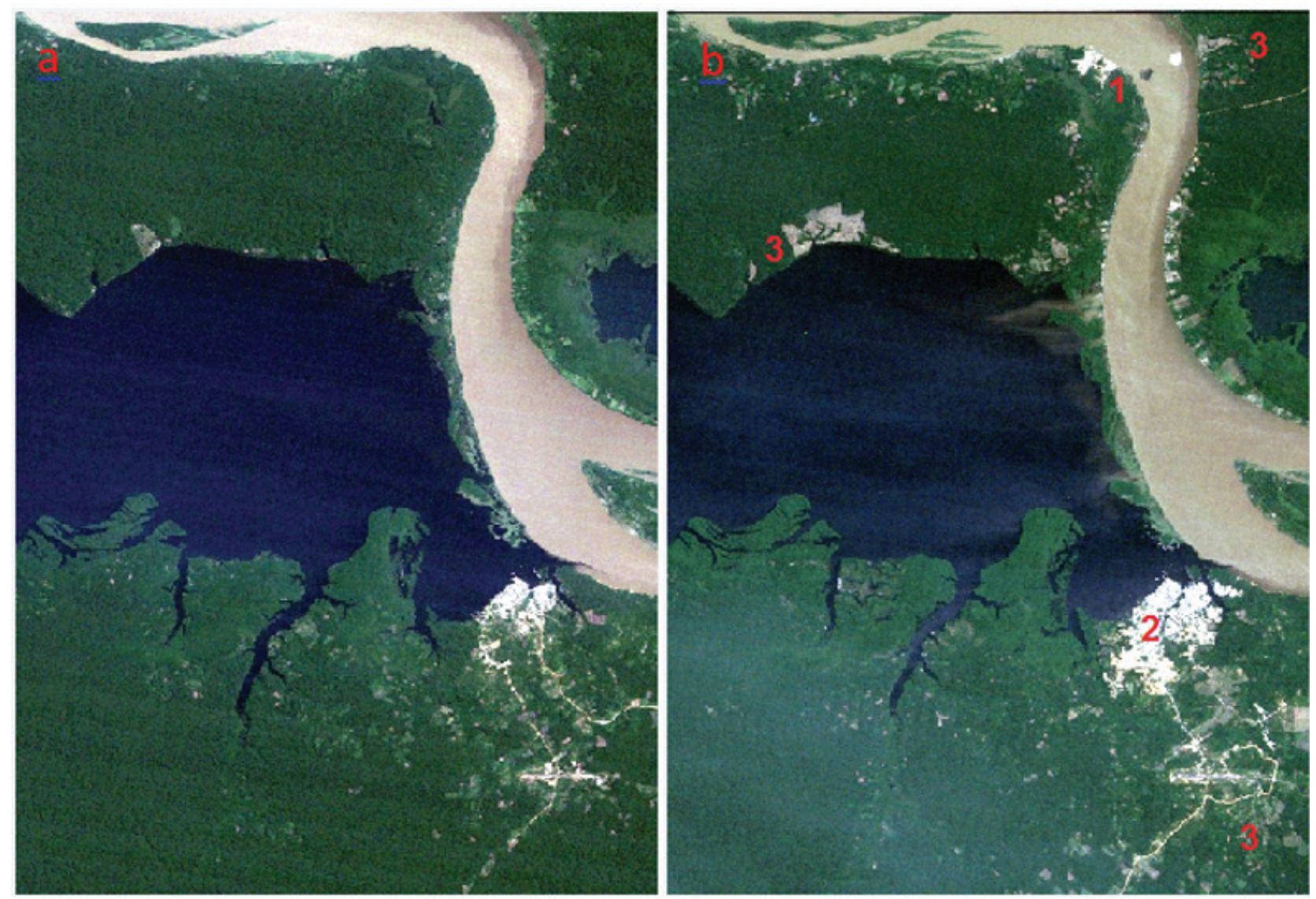

Org. dos autores.

(a) Imagem do dia 07/08/1986 e (b) Imagem do dia 08/08/2009. Terminal Solimões (1); solo exposto/cidade (2); regiões típicas de desflorestamento (3).

Comparando as Figuras 2(a) e 2(b), é possível visualizar a área onde foi construído o Terminal Solimões, o crescimento da zona urbana de Coari e algumas regiões de desflorestamento possivelmente consequência de atividade antropogênica. Um fato também observado foi a redução de algumas clareiras na porção sul, contrastando com um grande aumento deste tipo de cobertura na porção norte.

A partir das imagens do NDVI (Figura 3), foi obtido o histograma da distribuição de pixels de cada uma delas (Figura 4). Em seguida, foi estabelecida uma classificação da imagem (Figura 6), e determinada a porcentagem de uso do solo de cada classificação em cada uma das imagens (Figura 5). 
Uso de índice de vegetação para caracterizar a mudança no uso do solo em Coari-AM

Adriano Pereira Guilherme, Andréa Baima dos Santos Mota, Deniz dos Santos Mota, Nadja Gomes Machado, Marcelo Sacardi Biudes

Figura 3 - NDVI (com intensidade proporcional ao brilho do pixel) em região do município de Coari Amazonas/Brasil

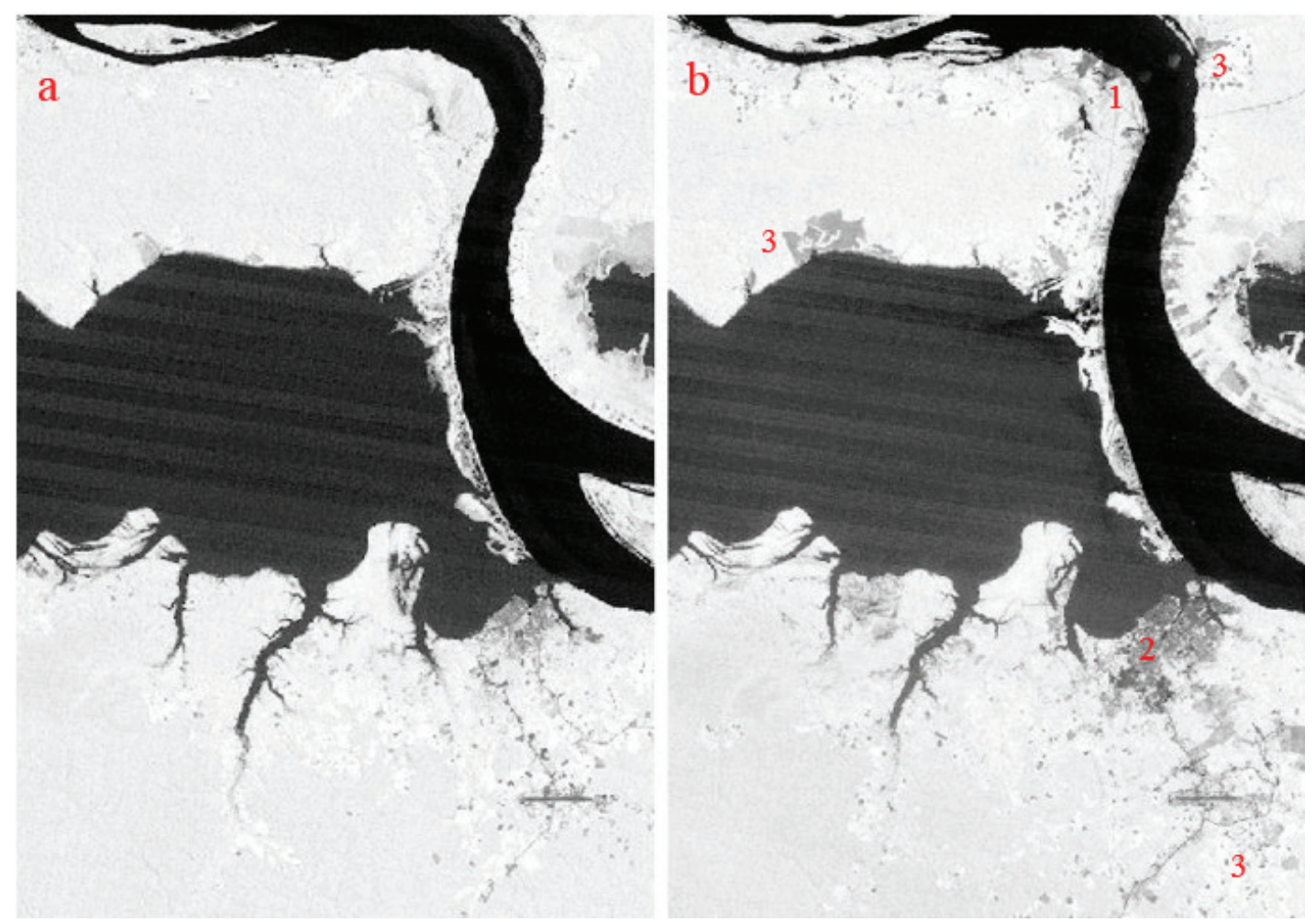

Org. dos autores.

(a) 1986 ; (b) 2009 .

Figura 4 - Histograma da distribuição de pixels por valores de NDVI e classes que serviram para a classificação quanto ao tipo de cobertura em região do município de Coari - Amazonas/Brasil.

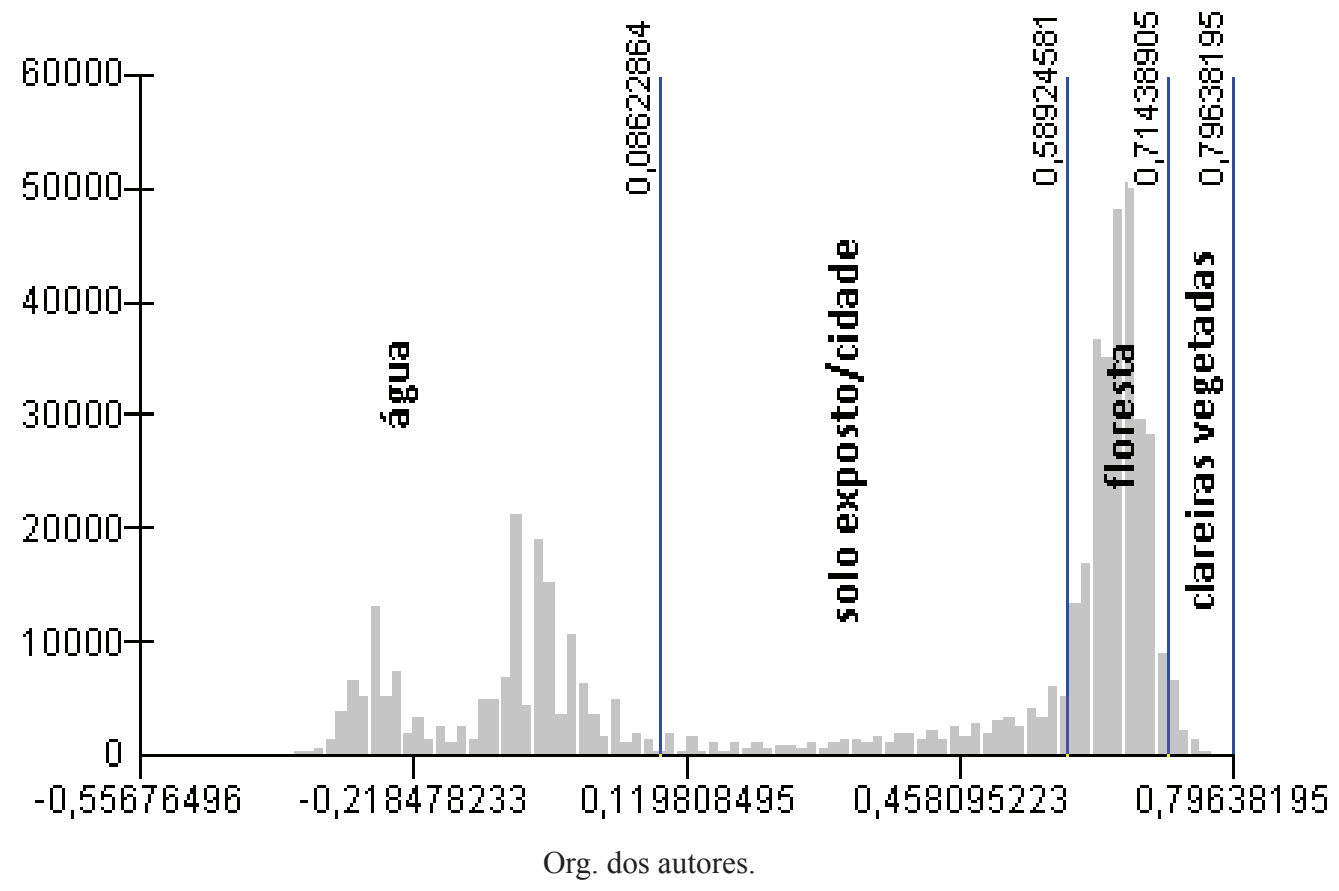

Soc. \& Nat., Uberlândia, 28 (2): 301-310, mai/ago/2016 
Uso de índice de vegetação para caracterizar a mudança no uso do solo em Coari-AM Adriano Pereira Guilherme, Andréa Baima dos Santos Mota, Deniz dos Santos Mota, Nadja Gomes Machado, Marcelo Sacardi Biudes

Figura 5 - Percentual de água, solo exposto/cidade, floresta e clareiras vegetadas nas imagens dos anos de 1986 e 2009 em região do município de Coari - Amazonas/Brasil.

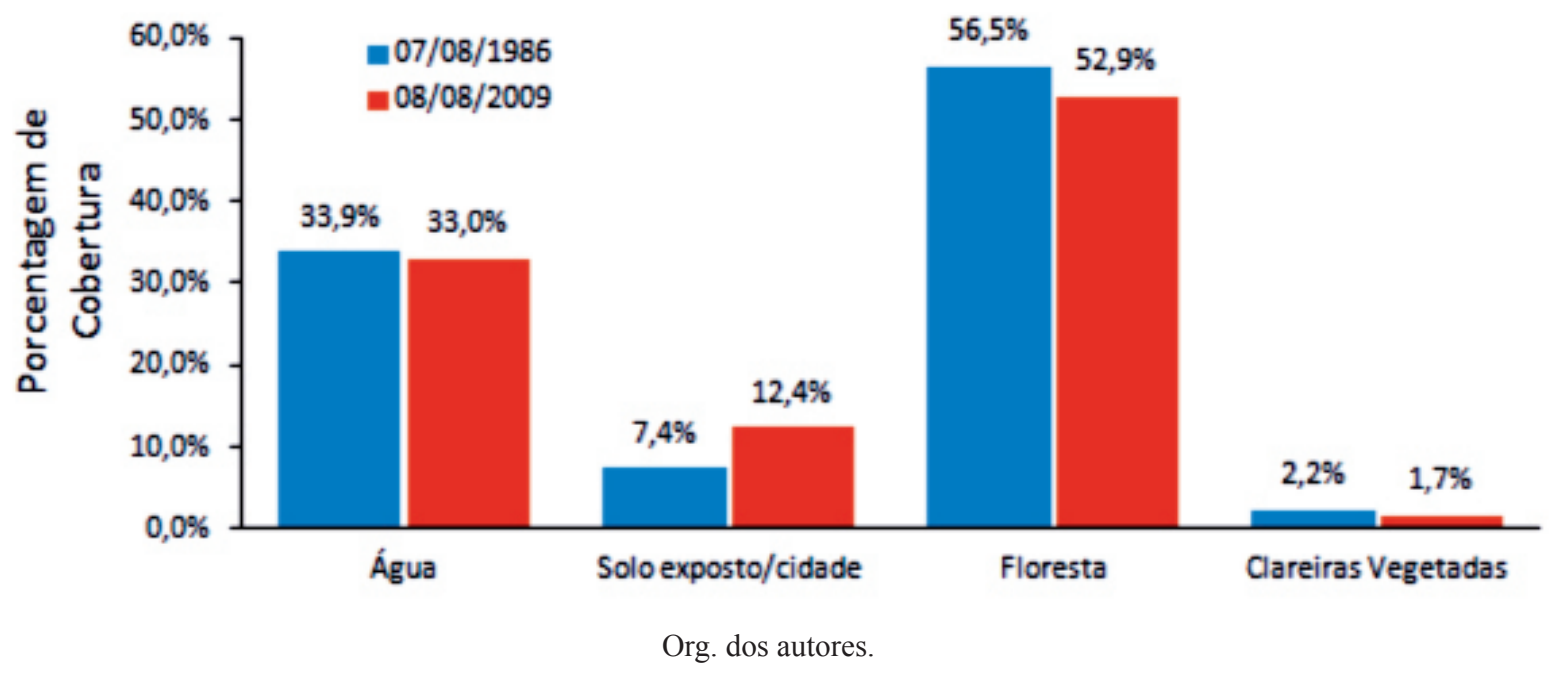

Tabela 2 - Estimativas para a média (intervalo de confiança de 95\%) e desvio padrão dos valores de NDVI para cada tipo de cobertura nas duas épocas analisadas em região do município de Coari-Amazonas/Brasil.

\begin{tabular}{|c|c|c|c|c|}
\hline COBERTURA & $\begin{array}{c}\text { NDVI médio (1986). IC } \\
\mathbf{9 5 \%}\end{array}$ & $\begin{array}{c}\text { NDVI médio } \\
\mathbf{( 2 0 0 9 ) . ~ I C ~ 9 5 \% ~}\end{array}$ & $\begin{array}{c}\text { Desvio Padrão } \\
\mathbf{( 1 9 8 6 )}\end{array}$ & $\begin{array}{c}\text { Desvio Padrão } \\
\mathbf{( 2 0 0 9 )}\end{array}$ \\
\hline Água & $(-0,1860 ;-0,1852)$ & $\begin{array}{c}-0,1186 ; \\
-0,1176)\end{array}$ & 0,0805 & 0,1036 \\
\hline Solo exposto/cidade & $(0,4341 ; 0,4369)$ & $(0,4178 ; 0,4200)$ & 0,2772 & 0,1433 \\
\hline Floresta & $(0,6567 ; 0,6569)$ & $(0,6529 ; 0,6531)$ & 0,1370 & 0,0285 \\
\hline Clareiras vegetadas & $(0,7298 ; 0,7303)$ & $(0,7274 ; 0,7279)$ & 0,0127 & 0,0118 \\
\hline
\end{tabular}

Org. dos autores.

Para estimar quantitativamente as alterações nas proporções dos tipos de cobertura, foi realizada uma breve análise estatística (Tabela 2) para os dois anos, onde também se determinou o percentual de água, solo exposto/cidade, floresta e clareiras vegetadas (Figura 5). Os intervalos de confiança para as médias do NDVI não se sobreponham para nenhum tipo de cobertura quando se comparam as duas épocas (Tabela 2), indicando que, mesmo em regiões intocadas pelo homem ou por eventos naturais, os valores estão diferindo significativamente.

A pequena variação do percentual de água deveu-se ao ciclo de cheia e vazante do rio. Observa-se também que uma parte significativa do que foi classificado como solo exposto/cidade se deve a fatores naturais, como o recuo das águas, o que resulta num solo sem cobertura vegetal. O ciclo do nível da água do rio é sazonal (registrando os maiores níveis no período de maio a julho), porém está sujeito às variações de intensidade e duração (MAGALHÃES; GOMES, 2013), o que justifica o aparecimento de porções de terra na imagem de 2009 que não aparecem na de 1986. As várzeas da região amazônica são ambientes totalmente dinâmicos, variando de formas e tamanhos devido ao processo natural das Terras Caídas, cujos fatores são associados com as propriedades físicas, químicas, mineralógicas e hidráulicas do solo que alteram todo o comportamento físico e mecânico do solo, provocando o desequilíbrio dos barrancos e o aparecimento de cicatrizes de Terras Caídas que por sua vez contribui para o processo de sedimentação (MAGALHÃES e GOMES, 2013). 
Uso de índice de vegetação para caracterizar a mudança no uso do solo em Coari-AM

Adriano Pereira Guilherme, Andréa Baima dos Santos Mota, Deniz dos Santos Mota, Nadja Gomes Machado, Marcelo Sacardi Biudes

A porcentagem de área de solo exposto/cidade aumentou entre 1986 e 2009 . O principal fator para esse aumento foi a expansão da zona urbana em função do aumento da população (ALMEIDA; SOUZA, 2008; IBGE, 2011) e ao aumento da atividade de extração dos recursos naturais (extração de madeira, óleo e gás natural). Outra atividade que merece atenção é a pesca na região. Em média, 51,3\% dos pescadores utilizam com maior frequência os lagos, seguido dos rios $(32,8 \%)$ e as fozes de rios (6,1\%) (INOMATA; FREITAS, 2011).

As porções classificadas como clareiras vegetadas visivelmente são zonas desmatadas que, no entanto, apresentam os maiores valores de NDVI. Isso se deve possivelmente a uma parcial recuperação da vegetação rasteira, com alta atividade fotossintética e refletividade, maior que nas zonas de florestas, as quais dispersam mais a radiação em virtude da arquitetura e "porosidade óptica" do dossel (PONZONI; REZENDE, 2004).

Algumas porções da região sudoeste da imagem de 2009 ficaram "salpicadas" de vermelho (Figura $6 \mathrm{~b})$, mesmo não havendo nenhum desmatamento visível. Possivelmente, nessas áreas houve invasões de "grilheiros", os quais são movimentos de fazendeiros que invadem áreas de forma ilegal. Essas práticas acontecem facilmente devido à sobreposição de terras demarcadas na Amazônia e pela descentralização dos cartórios. Os "grileiros" exploram madeiras e outros recursos naturais. O avanço dos "grileiros" resulta em grades áreas desmatadas com um número pequeno de pessoas (FEARNSIDE, 2007).

Figura 6 - Uso do solo obtido pelo índice de vegetação da diferença normalizada (NDVI) segundo as faixas de valores estabelecidos pela Figura 4 .

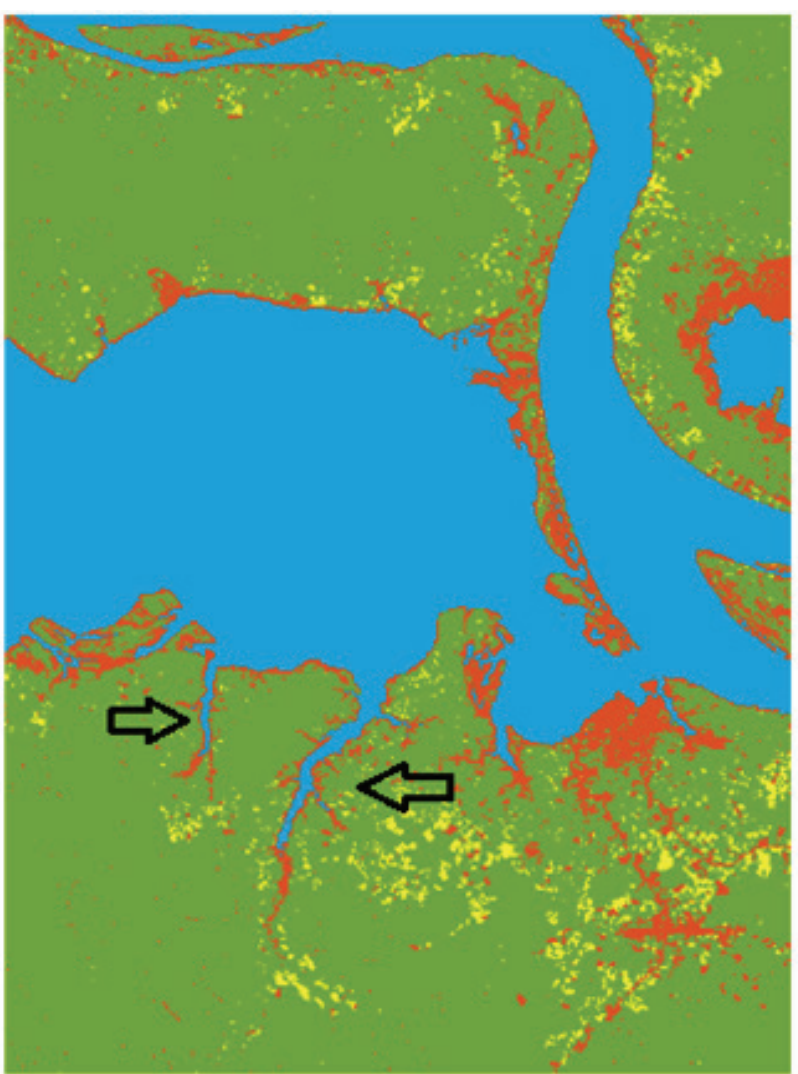

1986

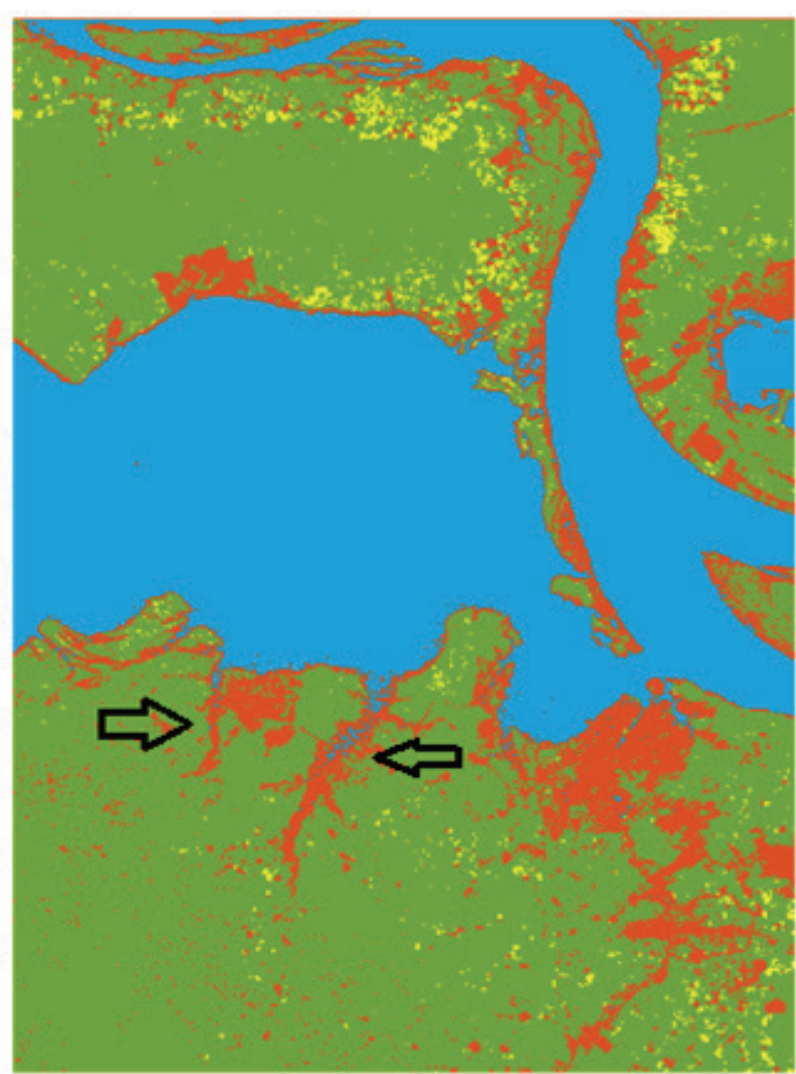

2009

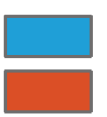

Água

Solo exposto/cidade
Floresta

Clareiras Vegetadas

Org. dos autores. 
Uso de índice de vegetação para caracterizar a mudança no uso do solo em Coari-AM Adriano Pereira Guilherme, Andréa Baima dos Santos Mota, Deniz dos Santos Mota, Nadja Gomes Machado, Marcelo Sacardi Biudes

\section{CONCLUSÕES}

A análise de imagens de satélite a partir do índice de vegetação de diferença normalizada (NDVI) mostrou-se uma importante ferramenta para a caracterização das mudanças de uso e ocupação do solo em Coari, Amazonas.

Os mapas de NDVI permitiram estabelecer um critério para classificar o tipo de cobertura da região estudada. Um rápido exame visual, evidenciado pelos números de uso e ocupação do solo, ilustrou o grande avanço da área urbana sobre a floresta nos arredores da cidade de Coari e regiões ribeirinhas.

Apesar de casos isolados de recuperação da floresta, o aumento das áreas desmatadas indicou aumento da atividade antropogênica nas últimas duas décadas, fato que pode ser explicado não apenas pelo aumento populacional, mas também pela grande dependência do extrativismo por parte da população no interior do Amazonas, estado que apresenta uma das maiores discrepâncias da atividade produtiva quando se considera a relação capital/interior.

\section{AGRADECIMENTOS}

A pesquisa foi apoiada pela Universidade Federal do Amazonas (UFAM), Instituto Federal do Amazonas (IFAM), Universidade Federal de Mato Grosso (UFMT), Programa de Pós Graduação em Física Ambiental (PPGFA/IF/UFMT), Conselho Nacional de Desenvolvimento Científico e Tecnológico (CNPq) [CNPq - Bolsas, Processo no 303625/2015-5], e Coordenação de Aperfeiçoamento de Pessoal do Ensino Superior (CAPES - Bolsas, Processo $n^{\circ}$ 9750/13-4 e $n^{\circ}$ 9768/13-0), e financiada pela Fundação de Amparo à Pesquisa do Estado de Mato Grosso (FAPEMAT PRONEM 2014, processo $n^{\circ}$ 561397/2014).

\section{REFERÊNCIAS}

AEROMAPA S/A, Cartografia, Informática e Projetos [Internet]. Disponível em: <http://www.aeromapa. com.br/pt/noticia/44-landsat-5-e-desativado-apos-mais-de-29-anos-em-orbita $>$. Acesso em: 01 set. 2014.
ALMEIDA, W.; SOUZA, N. Coari: Petróleo e Sustentabilidade - um exemplo amazônico. Desenvolvimento e Meio Ambiente, v.17, pp.69-92, 2008.

AMAZONAS, 2008. Secretaria de Estado de Planejamento e Desenvolvimento Econômico. Perfil Municipal - Coari (AM). Disponível em: <http://www. seplan.am.gov.br/planejamento /ddr/Condensadov3/ Conteudo/subregiao7/12-coari.html $>$. Acesso em: 01 set. 2014.

CHANDER, G.; MARKHAM, B. Revised Landsat-5 TM Radiometric Calibration Procedures and Postcalibration Dynamic Ranges. IEEE Transactions on Geoscience and Remote Sensing, v.41, n.11, 2003. DOI: 10.1109/TGRS.2003.818464

CUNHA, J.E.B.L; RUFINO, I.A.A; SILVA, B.B; CHAVES, L.B. Dinâmica da cobertura vegetal para a Bacia de São João do Rio do Peixe, PB, utilizando-se sensoriamento remoto, Revista Brasileira de Engenharia Agrícola e Ambiental, v. 16, n.5, pp.539-548, 2012. DOI: $10.1590 / \mathrm{S} 1415-43662012000500010$

ELVIDGE, C. D.; LYON, R. J. P. Influence of rock-soil spectral variation on the assessment of green biomass. Remote Sensing of Environment, v.17, pp.265-279, 1985. DOI: 10.1016/0034-4257(85)90099-9

FEARNSIDE, P. M. Brazil's Cuiabá-Santarém (BR163) Highway: the environmental cost of paving a soybean corridor through the Amazon. Environmental Management, v.39, pp.601-614, 2007. DOI: 10.1007/ s00267-006-0149-2

FEARNSIDE, P. M. The roles and movements of actors in the deforestation of Brazilian Amazonia. Ecology and Society, v.13, n.1, p.23, 2008.

FOODY, G. M. Assessing the accuracy of land cover change with imperfect ground reference data. Remote Sensing of Environment, v.114, pp.2271-2285, 2010. DOI: $10.1016 /$ j.rse.2010.05.003 
Uso de índice de vegetação para caracterizar a mudança no uso do solo em Coari-AM

Adriano Pereira Guilherme, Andréa Baima dos Santos Mota, Deniz dos Santos Mota, Nadja Gomes Machado, Marcelo Sacardi Biudes

GÓMEZ, C.; WHITE, J. C.; WULDER, M.A. Characterizing the state and processes of change in a dynamic forest environment using hierarchical spatio-temporal segmentation. Remote Sensing of Environment, v.115, pp.1665-1679, 2011. DOI: 10.1016/j.rse.2011.02.025

HOLBEN, B. N.; TUCKER, C. J.; FAN, C. J. Spectral assessment of soybean leaf area and leaf biomass. Photogrammetric Engineering and Remote Sensing, v.46, pp.651-656, 1980.

HOSTERT, P.; RODER, A.; HILL, J. Coupling spectral unmixing and trend analysis for monitoring of longterm vegetation dynamics in Mediterranean rangelands. Remote Sensing of Environment, v.87, pp.183-97, 2003. DOI: 10.1016/S0034-4257(03)00145-7

INOMATA, S. O.; FREITAS, C. E. C. Caracterização da frota pesqueira de Coari, Médio Solimões (Amazonas - Brasil). Revista Agroambiental, v.3, n.2, pp.65-70, 2011.

Instituto Brasileiro de Geografia e Estatística - IBGE. Disponível em: <http://cidades.ibge. gov.br/painel/ painel.php?codmun=130120>. Acesso em: 01 set. 2014.

IQBAL, M. An introduction to solar radiation. Library of Congress Cataloging in Publication Data. Canada: Academic Press, 1983.

KENNEDY, R. E.; ANDRÉFOUËT, S.; COHEN, W. B.; GÓMEZ, C.; GRIFFITHS, P.; HAIS, M.; HEALEY, S. P.; HELMER, E. H.; HOSTERT, P.; LYONS M. B.; MEIGS, G. W.; PFLUGMACHER, D.; PHINN, S. R.; POWELL, S. L.; SCARTH, P.; SEN, S.; SCHROEDER, T. A.; SCHNEIDER, A.; SNNENSCHEIN, R.; VOGELMAN, J. E.; WULDER, M. A.; ZHU, Z. Bringing an ecological view of change to Landsat-based remote sensing. Ecological Environment, v.12, n.6, pp.339-346, 2014. DOI: 10.1890/130066

MAGALHÃES, R. C.; GOMES, R. C. M. Mineralogia e química de solo de várzea e suas susceptibilidades no processo de terras caídas na comunidade do Divino Espírito Santo-AM. Sociedade \& Natureza, v.25, n.3, pp.6009-621, 2013.
MARKHAM, B. L.; BARKER, L. L. Thematic mapper bandpass solar exoatmospherical irradiances. International Journal of Remote Sensing, v.8, n.3, pp.517-523, 1987.DOI: 10.1080/01431168708948658

MENESES, P. R.; ALMEIDA, T. Introdução ao processamento de imagens de sensoriamento remoto. $\mathrm{UnB} / \mathrm{CNPq}, 2012$.

MOURÃO, G. M. N. Colonización reciente y asentamientos rurales en el sureste de Roraima, Amazonia Brasileña: entre la política y la naturaleza. 2003. $480 \mathrm{f}$. Tese de doutorado, Universidad de Valladolid, Espanha. 2003.

MOURÃO, R.; RIVAS, A.; FRAXE, T. O Estado da economia nas comunidades de várzea: atividades tradicionais e integração de mercado, pp. 149-170, 2007. In: Teixeira, P.; Brasil, M.; Rivas, A. (Eds). Produzir e viver na Amazônia Rural: estudo sociodemográfico de comunidades do Médio Solimões. Manaus: EDUA.

PEEL, M. C.; FINLAYSON, B. L.; MCMAHON, T. A. Updated world map of the Köppen-Geiger climate classification. Hydrology and Earth System Sciences, v.11, n.S1, pp.1633-1644, 2007. DOI: 10.5194/hess11-1633-2007

PETROBRAS. Urucu. Disponível em: <sites.petrobrás.com.br/minisite/urucu/urucu.html $>$. Acesso em: 01 set. 2014.

PONZONI, F. J.; REZENDE, C. P. Caracterização espectral de estágios sucessionais de vegetação secundária arbórea em Altamira (PA), através de dados orbitais. Revista Árvore, v.28, n.4, pp.535-545, 2004. DOI: $10.1590 / \mathrm{S} 0100-67622004000400007$

TUCKER, C. J., Red and photographic infrared linear combinations for monitoring vegetation. Remote sensing of Environment, v.8, pp.325-348, 1985. DOI: 10.1016/0034-4257(79)90013-0 